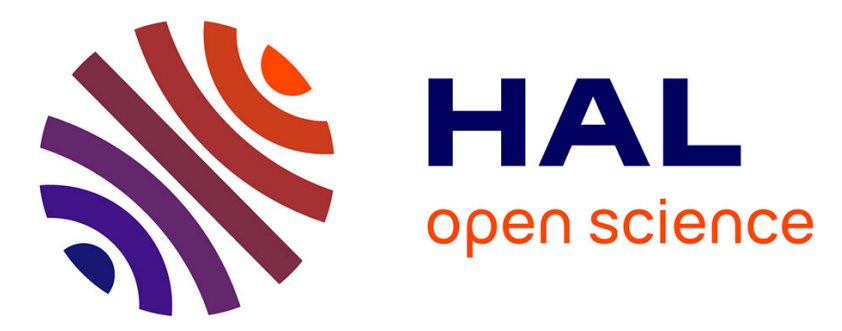

\title{
Vibro-Acoustic Modeling of a Trimmed Truck Cabin in Low Frequency Range to Tackle the Challenge of Weight Reduction
}

Fabien Acher, Kerem Ege, Céline Sandier, Youssef Gerges

\section{- To cite this version:}

Fabien Acher, Kerem Ege, Céline Sandier, Youssef Gerges. Vibro-Acoustic Modeling of a Trimmed Truck Cabin in Low Frequency Range to Tackle the Challenge of Weight Reduction. 10th International Styrian Noise, Vibration \& Harshness Congress: The European Automotive Noise Conference, Jun 2018, Graz, Austria. pp.2018-01-1549, 10.4271/2018-01-1549 . hal-01829410

\section{HAL Id: hal-01829410 https://hal.science/hal-01829410}

Submitted on 18 Jul 2018

HAL is a multi-disciplinary open access archive for the deposit and dissemination of scientific research documents, whether they are published or not. The documents may come from teaching and research institutions in France or abroad, or from public or private research centers.
L'archive ouverte pluridisciplinaire HAL, est destinée au dépôt et à la diffusion de documents scientifiques de niveau recherche, publiés ou non, émanant des établissements d'enseignement et de recherche français ou étrangers, des laboratoires publics ou privés. 


\section{Vibro-Acoustic Modeling of a Trimmed Truck Cabin in Low Frequency Range to Tackle the Challenge of Weight Reduction}

Fabien Acher, Volvo GTT (France)

Kerem Ege, Céline Sandier, Youssef Gerges, INSA Lyon / LVA

Citation: Acher, F., Ege, K., Sandier, C., and Gerges, Y., "Vibro-Acoustic Modeling of a Trimmed Truck Cabin in Low Frequency Range to Tackle the Challenge of Weight Reduction," SAE Technical Paper 2018-01-1549, 2018, doi:10.4271/2018-01-1549 


\title{
Vibro-acoustic modeling of a trimmed truck cabin in low frequency range to tackle the challenge of weight reduction
}

\author{
Author, co-author (Do NOT enter this information. It will be pulled from participant tab in \\ MyTechZone) \\ Affiliation (Do NOT enter this information. It will be pulled from participant tab in MyTechZone)
}

\begin{abstract}
In the challenge of reducing the weight of the vehicle structures, a particular focus has to be done on the interior noise. Indeed, the weight reduction of the structure often implies an increase of the noise in the cabin.

To maintain a constant acoustic performance, acoustic packages often have to be added, the challenge being that the weight of the acoustic materials added remains lower than the weight saved in the structure.
\end{abstract}

In today's engineering world, numerical simulation is the primary tool to assess the vibro-acoustic behavior of the vehicle during the design phase. To tackle the challenge of weight reduction, it is necessary to simulate accurately the vibro-acoustic response of the structure including the acoustic treatments.

This paper presents the validation of a simulation method for the vibro-acoustic response of a truck cabin, taking into account the effect of acoustic treatments, in the frequency range [0-200Hz]. The method combined a modal scheme for the structure and the cavity with a physical scheme for the acoustic treatment (porous materials).

The model consists in a truck cabin with its cavity and five acoustic treatments (three floormats, the headliner and the rear trim panel). A measurement campaign is performed to get reference NTFs, VTFs and local inertances. The model without any treatments is first correlated. The noise reduction given by each treatment alone as well as grouped together is simulated. The comparisons between the simulated and measured results show good agreement both in term of spectrum and amplitude. Although some discrepancies in the low frequency range remain unexplained, the method is considered as validated.

\section{Introduction}

In 2012-2015, the CLIC project (City Lightweight and Innovative $\mathrm{Cab}$ ), a scientific collaboration gathering public and private organizations, was carried out to propose an innovative lighten truck cabin with the use of high-tensile steels.

During this project, a particular focus was put on the acoustic analysis of the cabin. An important part of the project was dedicated to develop and validate numerical methods to assess the vibroacoustic behavior of a structure including the acoustic treatments (both for low and $\mathrm{mid} /$ high frequency ranges [1]).

Page 1 of 8
In the low frequency range (typically below $300 \mathrm{~Hz}$ ), simulations of body-in-white structures coupled to cavities have been performed for several decades using the finite element method in modal coordinates. These simulations generally do not take into account the effect of the acoustic treatments. However, as shown in this paper, their influence can be significant on the noise level.

To close this gap, a numerical method for the vibro-acoustic response $[0-200 \mathrm{~Hz}]$ of the trucks cabin taking into account the effect of the acoustic treatments has been implemented. This paper summarizes the works done to correlate this method.

Possible numerical strategies are shortly reviewed. The strategy chosen for implementation is detailed. The measurement campaign performed to validate the method is introduced as well as the details of the FEM models. Finally, results are shown and comparisons between tests and simulations are discussed for various indicators (VTF/NTF/Noise reduction).

\section{Numerical Strategy}

In the automotive/truck industry, the FEM simulation a structure below $300 \mathrm{~Hz}$ (body-in-white for example), coupled or not with a cavity, is generally done using modal methods (a modal extraction and a modal frequency response). Considering the number of degrees of freedom of the models (several millions), a direct approach, inverting the matrix in physical coordinates, is not realistic with today's computational resources. The modal approach is now well established and has demonstrated for several decades its ability to simulate accurately this kind of lightly damped problems.

A typical truck cabin structure is trimmed with acoustic treatments whose main function is to bring acoustic absorption in the cabin (trim panels), insulate from exterior noise (floormats and trim panels) or reduce the vibrations of the cabin floor (floormats). The materials of these treatments have frequency dependant properties and are generally heavily damped. For these reasons, it is difficult to represent them with modal methods.

To overcome this limitation several methods have been proposed:

- A first group of methods based on super-elements reduction techniques is mentioned [2]. The important computational resources associated (time and storage space) are a strong limitation for these methods. 
- A second method, based on the energetic behaviour is proposed [3]. In this method, each mode can be affected by the acoustic components but the effect on the coupling between the modes is neglected.

- $\quad$ A third method, described in [2] and [4] consists in condensing the impedance matrix of the acoustic treatments to its degrees of freedom interfaced with the structure or the cavity. The condensed impedance matrix is then projected into the modal space and injected in the modal impedance matrix of the complete system. This $3^{\text {rd }}$ method introduces coupling terms between the modes and overcomes the limitation of the $2^{\text {nd }}$ one.

This $3^{\text {rd }}$ method, based on reduced impedance matrices of the trim components is chosen and implemented in the present work.

\section{Theoretical Framework}

The theoretical development of the approach based on reduced impedance matrices is presented.

The dynamic behaviour of a system made of a structure (such as the body-in-white) and its cavity, represented in modal coordinates can be written as:

$\left(\begin{array}{cc}Z_{S}(\omega) & C_{S F} \\ C_{S F}^{T} & Z_{F}(\omega)\end{array}\right) \cdot\left(\begin{array}{c}\alpha_{S}(\omega) \\ \alpha_{F}(\omega)\end{array}\right)=\left(\begin{array}{c}\Phi_{S}^{T} \cdot F_{S} \\ \frac{\Phi_{F}^{T} \cdot F_{F}}{\omega^{2}}\end{array}\right)$

With

$\left\{\begin{array}{c}Z_{S}(\omega)=K_{S}+\frac{i}{\omega} C_{S}-\omega^{2} M_{S} \\ Z_{F}(\omega)=\frac{1}{\omega^{2}} K_{F}+\frac{i}{\omega} C_{F}-\omega^{2} M_{F}\end{array}\right.$

$\omega$ is the pulsation; $K, C$ and $M$ are the modal stiffness, modal damping and modal mass matrices for the structure and cavity. $C_{S F}$ is the modal structure-fluid coupling matrix. $F$ are the excitations applied on the structure and the cavity.

An acoustic treatment $T$, such as the floormat represented Figure 1 is coupled to the structure through its lower surface $\left(\Gamma_{S}\right)$ and to the cavity through its upper surface $\left(\Gamma_{F}\right)$.

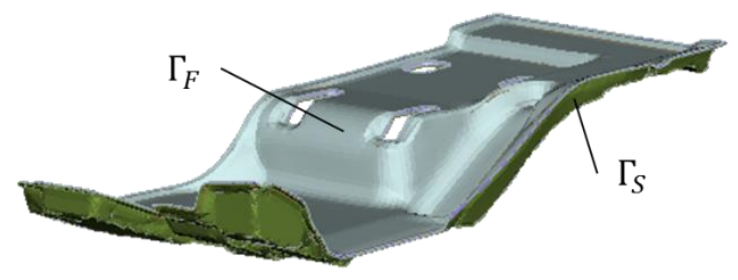

Figure 1: View of a floormat

The degrees of freedom of its finite element model can be separated into inner degrees of freedom $\left(D_{\text {inner }}\right)$ and coupling degrees of freedom $\left(D_{\Gamma}\right)$, located on the surfaces $\Gamma_{s}$ and $\Gamma_{F}$. Its impedance matrix in physical coordinates can be written:

$Z_{T}(\omega)=\left(\begin{array}{cc}Z_{\text {inner }}(\omega) & Z_{\text {inner }, \Gamma}(\omega) \\ Z_{\Gamma, \text { inner }}(\omega) & Z_{\Gamma}(\omega)\end{array}\right)$
In physical coordinates, its dynamic behaviour is described by:

$$
\left(\begin{array}{cc}
Z_{\text {inner }}(\omega) & Z_{\text {inner }, \Gamma}(\omega) \\
Z_{\Gamma, \text { inner }}(\omega) & Z_{\Gamma}(\omega)
\end{array}\right) \cdot\left(\begin{array}{c}
D_{\text {inner }}(\omega) \\
D_{\Gamma}(\omega)
\end{array}\right)=\left(\begin{array}{c}
0 \\
h_{\Gamma}
\end{array}\right)
$$

Where $h_{\Gamma}$ are the excitation applied on the coupling surfaces (there is no excitation on the inner degrees of freedom).

Developing this system, it is possible to describe the inner degrees of freedom in function the degrees of freedom of its interfaces with the structure and the cavity. The dynamic behaviour of the treatment can then be reduced to its interface degrees of freedom:

$$
\underbrace{\left(Z_{\Gamma}(\omega)-Z_{\Gamma, \text { inner }}(\omega) \cdot Z_{\text {inner }}^{-1}(\omega) \cdot Z_{\text {inner }, \Gamma}(\omega)\right)}_{Z_{\text {red }}} \cdot D_{\Gamma}(\omega)=h_{\Gamma}
$$

This reduced impedance matrix $Z_{\text {red }}$ is then projected into the structure and cavity modes and injected into equation (1).

$$
Z_{\text {red }}(\omega)=\left(\begin{array}{cc}
\Phi_{S}^{T} & 0 \\
0 & \Phi_{F}^{T}
\end{array}\right) \cdot Z_{\text {red }}(\omega) \cdot\left(\begin{array}{cc}
\Phi_{S} & 0 \\
0 & \Phi_{F}
\end{array}\right)
$$

$$
\left(\begin{array}{cc}
Z_{S}(\omega)+z_{r e d, S}(\omega) & C_{S F}+z_{r e d, C}(\omega) \\
C_{S F}{ }^{T}+z_{r e d, C}{ }^{T}(\omega) & Z_{F}(\omega)+z_{r e d, F}(\omega)
\end{array}\right) \cdot\left(\begin{array}{c}
\alpha_{S}(\omega) \\
\alpha_{F}(\omega)
\end{array}\right)=\cdots
$$

$$
=\left(\begin{array}{c}
\Phi_{S}^{T} \cdot F_{S} \\
\frac{\Phi_{F}^{T} \cdot F_{F}}{\omega^{2}}
\end{array}\right)
$$

The "updated" system is then solved to retrieve the vibro-acoustic response including the effect of the acoustic treatment.

The computation of the reduced impedance matrix is the "costly" step since it needs the inversion of a dense matrix. The computational cost will increase with the size of the treatment.

In this method, it is considered that the presence of the acoustic treatment will not change the mode shapes or, in other words, that those mode shapes can be used as a mathematical basis for the representation of the system response.

\section{Simulation Model}

The structure, the cavity and the acoustic treatments of the truck cabin are modelled with FEM method.

\section{Structure}

The structure model corresponds to the body-in-white of the structure with the windows. The system that decouples the cabin from the ground as well as the adaptations performed on the cab to close it completely are included in the model (see Figure 5 Error! Reference source not found.). Only the roof hatch is simplified to a local mass applied on its connection points, its vibro-acoustic coupling with the cavity is not considered. The damping treatments made of bituminous pads are included in this model. A view of the finite element mesh of the cabin structure can be seen Figure 2.

Page 2 of 8 


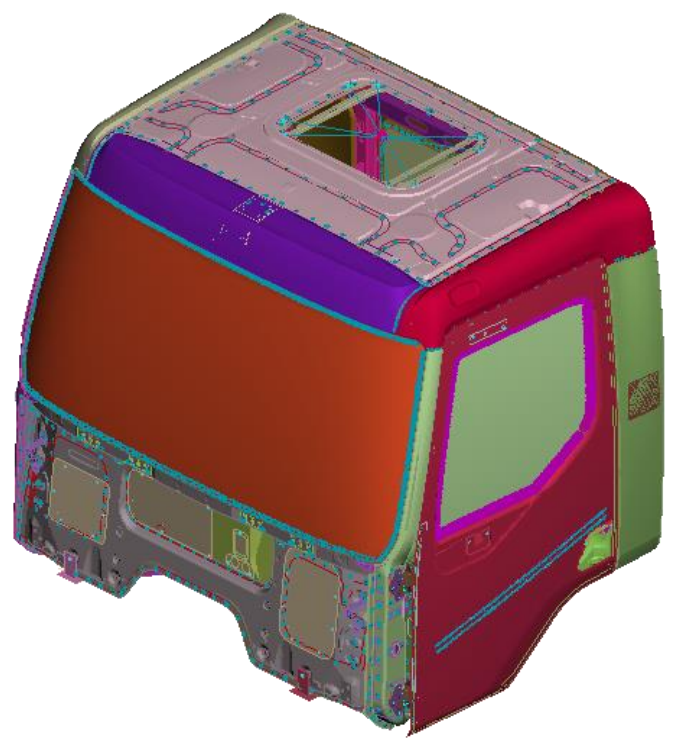

Figure 2: View of the cabin structural model

\section{Cavity}

The volume of the cavity varies depending on the acoustic treatments present. For each configuration of treatments, the exact cavity volume is meshed. An average mesh size of $35 \mathrm{~mm}$ is used to ensure the quality of the modal extraction up to $900 \mathrm{~Hz}$. The cavity mesh without any acoustic treatment is shown Figure 3.

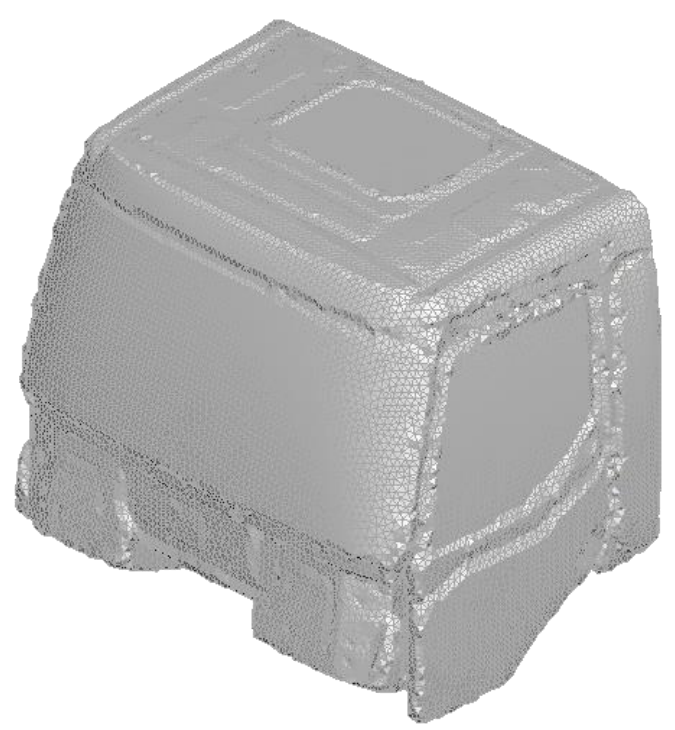

Figure 3: View of the cavity mesh for the bare configuration

\section{Acoustic treatments}

The acoustic treatments considered are the floormats (made of one layer of polyurethane foam and one heavy layer of rubber material) and the trim panels (made of compressed felt, covered with a nonwoven scrim and partially reinforced with an absorbent felt). They are divided in five different parts (see Figure 4):

- $\quad$ the headliner;

- the rear trim panel;

- the driver floormat;

- the tunnel floormat;

- the passenger floormat.

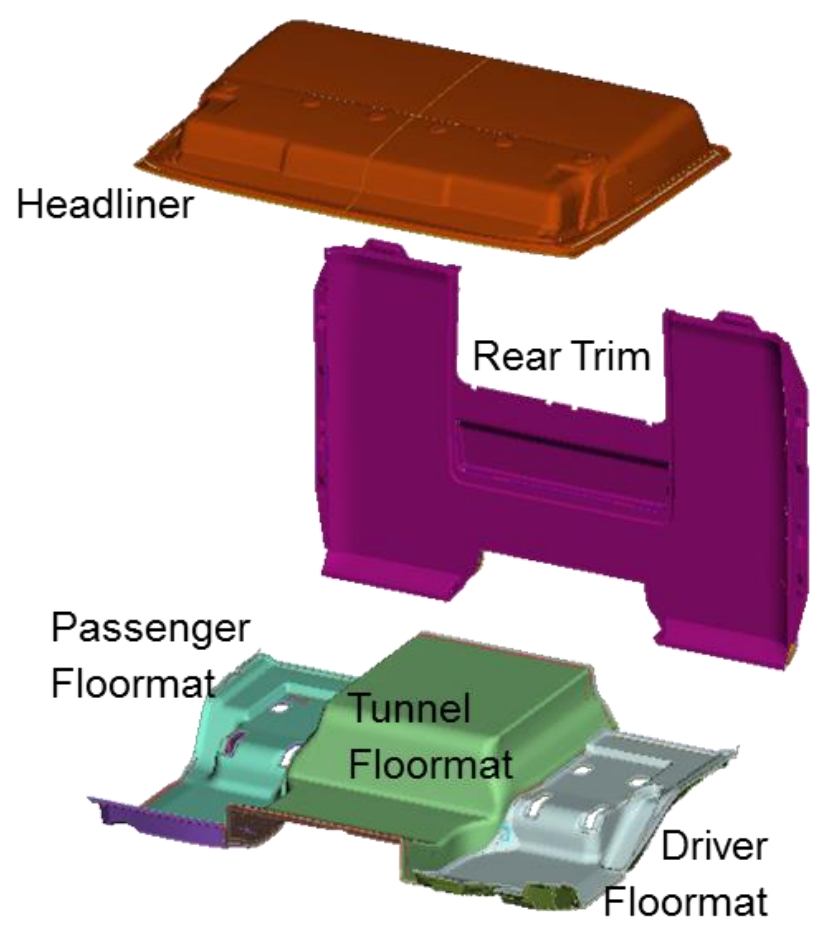

Figure 4: View of the 5 acoustic treatments

They are meshed with standard meshing requirements. To avoid continuity of displacement between the structure and the treatments (which does not happen in the reality) an air gap is modelled between them. The porous and fibrous components models rely on the Biot theory. The Johnson-Champoux-Allard model is used [5],[6].

This model requires specific material properties, including the resistivity, the porosity, the tortuosity, the viscous length and the thermal length. Their mechanical properties (Young modulus, Poisson's ratio, loss factor) are also needed for an accurate modelling.

A proper characterization of all the materials is performed. The air flow resistivity is measured following the standard ISO-9053. The porosity is measured with a "porosimeter" following the method described in [7]. A third measurement in a Kundt tube allows determining the remaining acoustic parameters from their analytical expression in the Johnson-Champoux-Allard model [5],[6]. 


\section{Simulation Process:}

The calculation needs several steps that must be executed sequentially:

1. In a first step, the modes of the cavity and the modes of the structure are extracted. The maximum frequency of extraction is taken high enough to avoid modal truncature effect. The modes shapes are stored on disk. The modal damping matrix of the structure, including the effect of the bituminous damping pads, is also extracted and stored on disk. The MSC Nastran software package is used for this step. The Actran software package is used to perform steps 2 to 4 .

2. In a second step, for each treatment the impedance matrices are reduced to the degrees of freedom coupled with the structure and cavity. They are immediately projected into the modal spaces and stored on disk.

3. In a third step, the fluid / structure coupling in areas where no acoustic components are present is computed. When acoustic components are present, the fluid/structure coupling is contained on the reduced impedance matrices.

4. As a final step, the modal frequency response of the complete system is assembled and solved for the given excitations.

\section{Measurements Campaign}

The measurement campaign is performed to acquire data to be compared with simulation results.

In the measurement set-up the cabin is decoupled from its support using air springs fixed at cab anchorages (see Figure 5Error! Reference source not found.). The air springs provide a decoupling frequency below $5 \mathrm{~Hz}$. All holes (cables pass-through, steering column hole...) were capped to ensure a proper acoustic sealing of the cavity (see Figure 5Error! Reference source not found.).

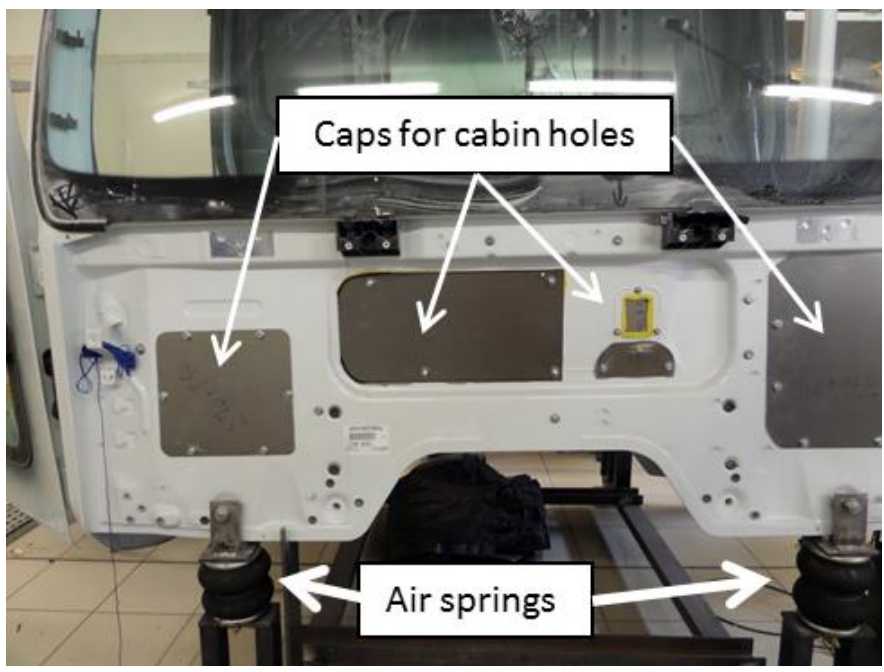

Figure 5: View of the decoupling system and the caps to close the cavity
A set of 18 microphones are placed inside the cab on different locations, two of them being positioned at the driver's ears (see Figure 6Error! Reference source not found.). A high number of microphones allow computing an average pressure within the cavity.

Several accelerometers are positioned on the cab structure, mainly at the cab anchorages, on the floor areas and on the rear wall ( 2 of them are displayed Figure 7Error! Reference source not found.).

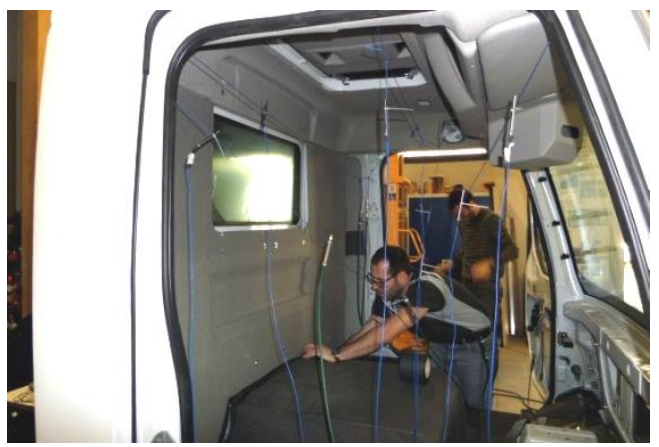

Figure 6: View of the microphones positionned inside the cabin

The cabin is excited using an impact hammer in the normal direction to the surface close to all accelerometers. For each excitation, all transfer functions are measured including:

- Local inertance;

- Vibration Transfer Functions to all accelerometers;

- $\quad$ Noise Transfer Functions to all microphones.

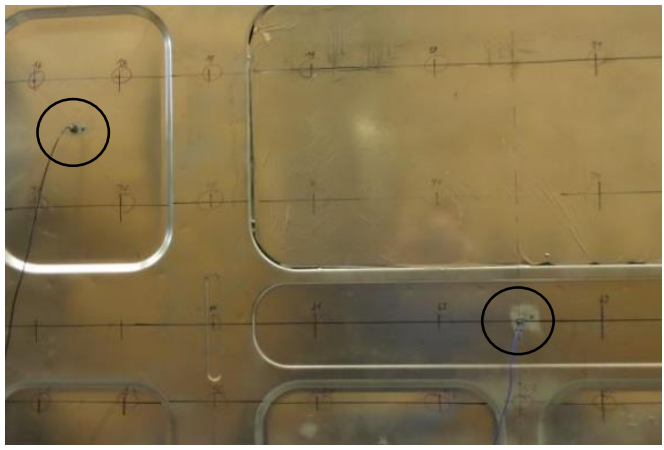

Figure 7: View of the accelerometers positionned on the rear wall of the cabin

Different configurations are measured in order to determine the influence of the acoustic treatments, both individually and grouped:

- Configuration 1: cabin with all acoustic treatments (floormats, rear trim panel and headliner);

- Configuration 2: cabin with floormats and rear trim panel (without headliner);

- Configuration 3: cabin with floormats only;

- Configuration 4: cabin with rear trim panel only;

- Configuration 5: cabin without any treatment.

One of the main remarks from the measurement is that the effect of the acoustic treatments occurs above $50 \mathrm{~Hz}$ (see Figure 8 Error! Reference source not found.). 


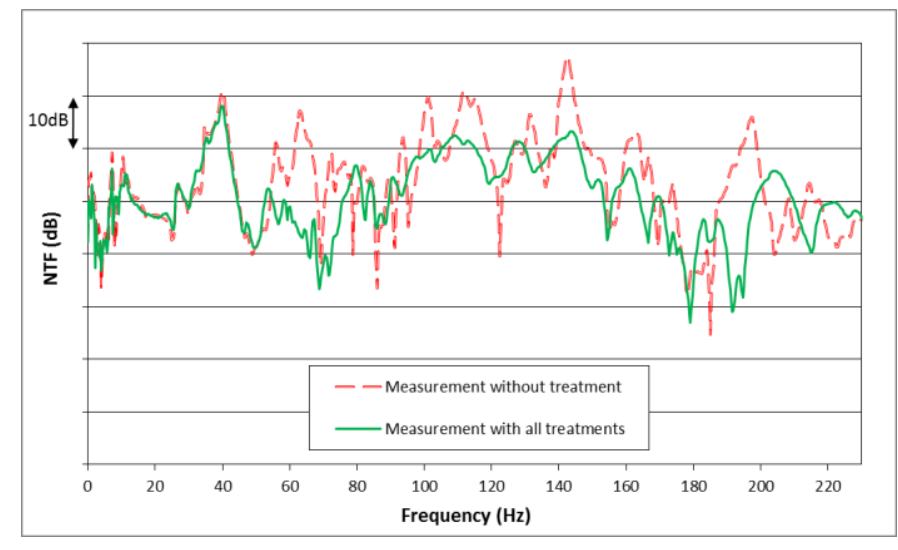

Figure 8: Effect of the acoustic treatment on the NTF.

The use of 18 microphones allows measuring the mean pressure within the cavity. To validate that 18 microphones are enough to measure the mean pressure a simulation is performed on the configuration without treatment. The mean pressure is computed and compared using all the cavity volume and the 18 microphones. Results are presented Figure 9Error! Reference source not found. Results are very close and around $1 \mathrm{~dB}$ difference maximum is observed. The measurement of the mean NTF from 18 microphone is validated.

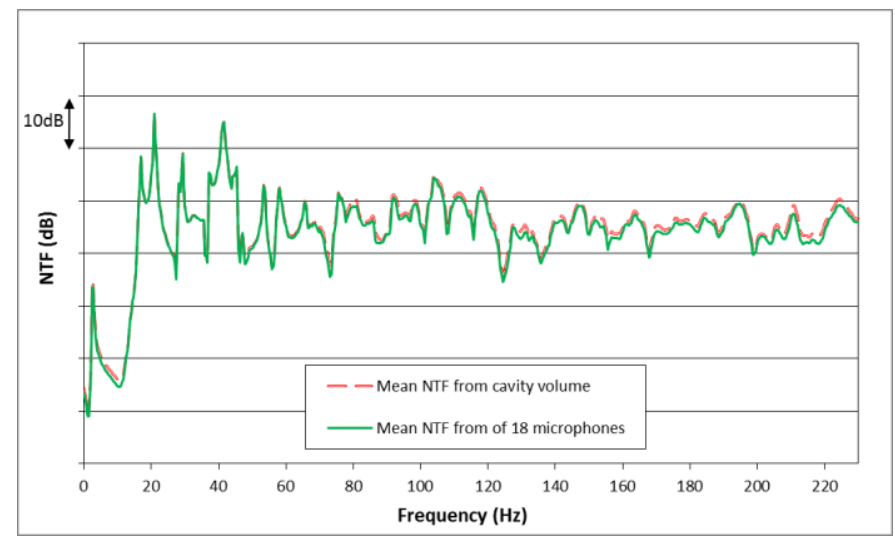

Figure 9: Comparison of mean NTF obtained from the cavity volume and from 18 microphones

\section{Results and Discussions}

Different indicators are looked at. They are listed with an increasing complexity:

- The inertance at the anchorage points of the cabin on the chassis or at the centre of the panels of the cabin;

- $\quad$ The VTF between the anchorage points and the panel points;

- The NTF between the panel points and the driver's ears;

- $\quad$ The NTF between the cab anchorage points and the driver's ears.

The results are analysed up to $230 \mathrm{~Hz}$ which corresponds to the maximum frequency excited by the $2^{\text {nd }}$ harmonic of the firing order of the engine ( $3^{\text {rd }}$ order for a 6-cylinders engine). Among all the results available, a few are presented to illustrate the conclusions issued from their analysis. Three excitation points are considered as shown Figure 10.

A first one close to the front left anchorage point of the cabin on the chassis (excitation in transversal direction), a second one on the tunnel area below the tunnel floormat (excitation in vertical direction) and a third one on the rear panel behind the rear trim panel (excitation in longitudinal direction). The NTF are given to the driver's left ear.

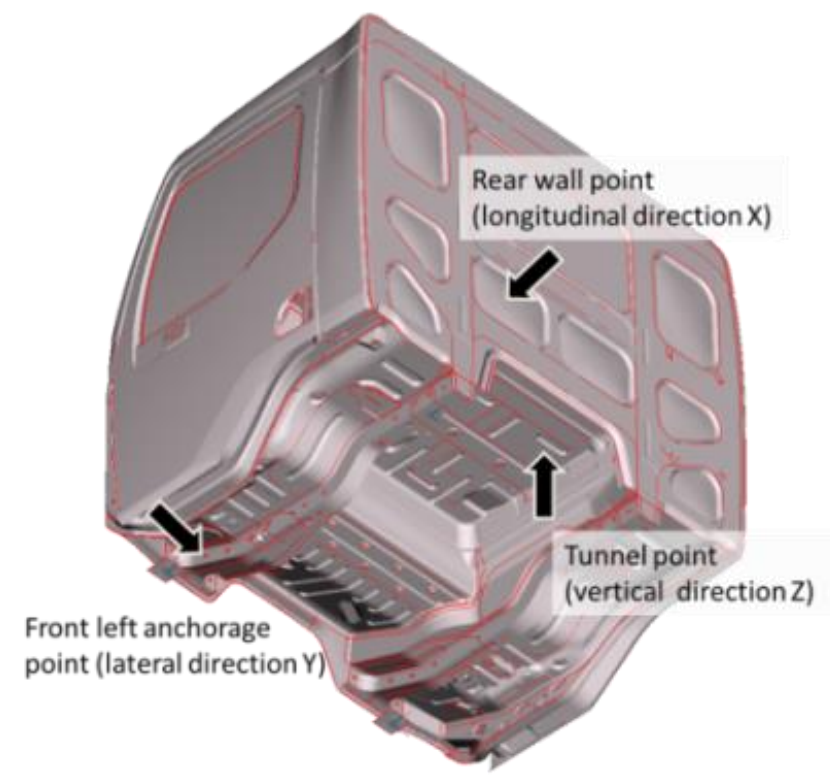

Figure 10: View of the excitation points for the results presented

\section{Correlation of the bare model}

The vibro-acoustic response of the bare model, without any acoustic treatment, is simulated and compared to the measurements.

The correlation work does not aim at artificially modifying local parameters or setting non-physical materials to exactly match the measurement on the whole frequency range. It rather consists in modifying global parameters (junction modelling, material young modulus...) to identify and bring closer the global or panel modes of the structure. The goal is to assess that the simulation represents well the measurements. At the end of the correlation, the remaining shifts between the simulation and experimental results shall be known and understood.

Examples of final comparisons for inertance results are shown in Figure 11 for the front left anchorage point and Figure 12 for the rear wall point.

Correlation is satisfying both in term of spectrum content and levels.

\section{Page 5 of 8}




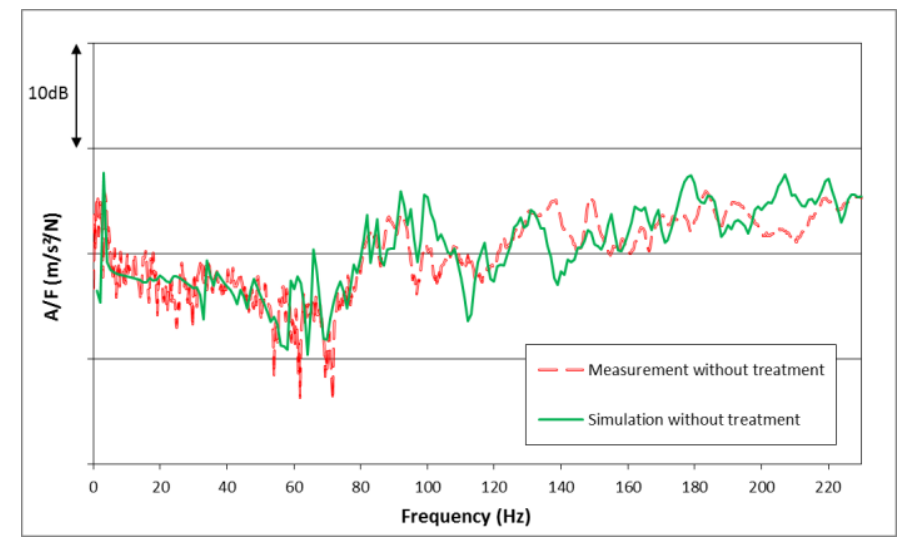

Figure 11: Measured and simulated inertance at the front left anchorage point

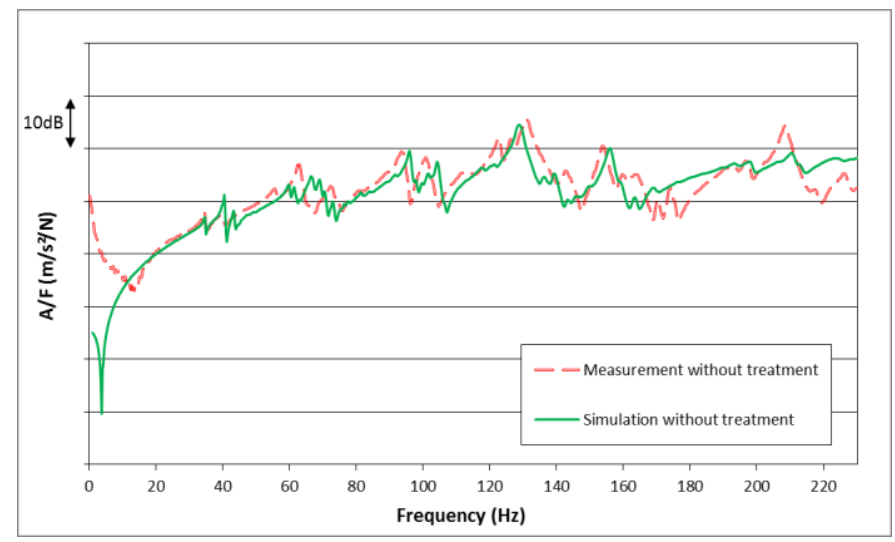

Figure 12: Measured and simulated inertance at a point of the rear wall panel

The VTF between the anchorage points and the centres of panels is then analysed to assess the correlation. Example of VTF correlation between the front anchorage point and the rear wall point is displayed Figure 13.

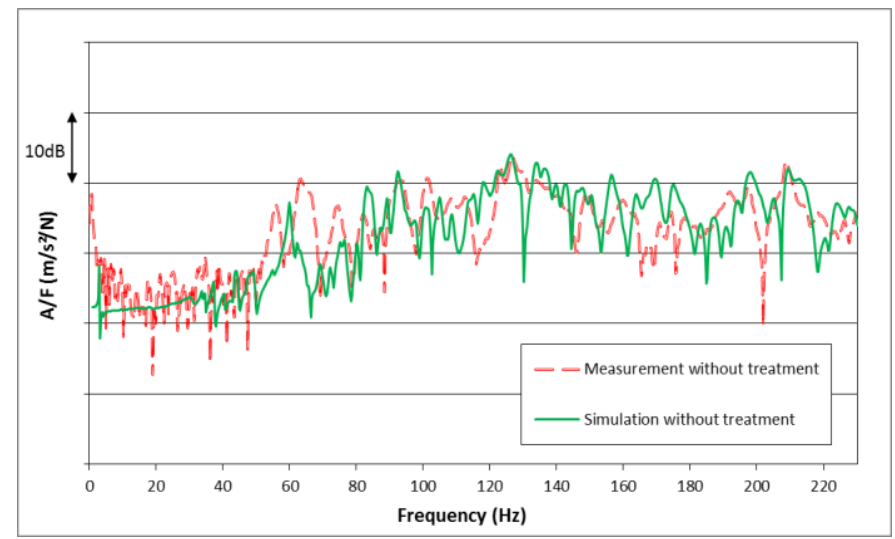

Figure 13 Measured and simulated VTF between the front left anchorage point and a point on the rear wall

The simulated and measured VTFs show similar spectrum and levels. The differences are mainly inherited from the differences observed on the local inertances.

Page 6 of 8
Finally the measured and simulated NTFs are compared. Figure 14 and Figure 15 show NTFs to driver's ear from the front left anchorage point and from the rear wall point respectively.

The good agreement on the structure response is similarly observed on the acoustic response. Results from the mean NTF (average of NTF from 18 microphones) have similar level of correlation.

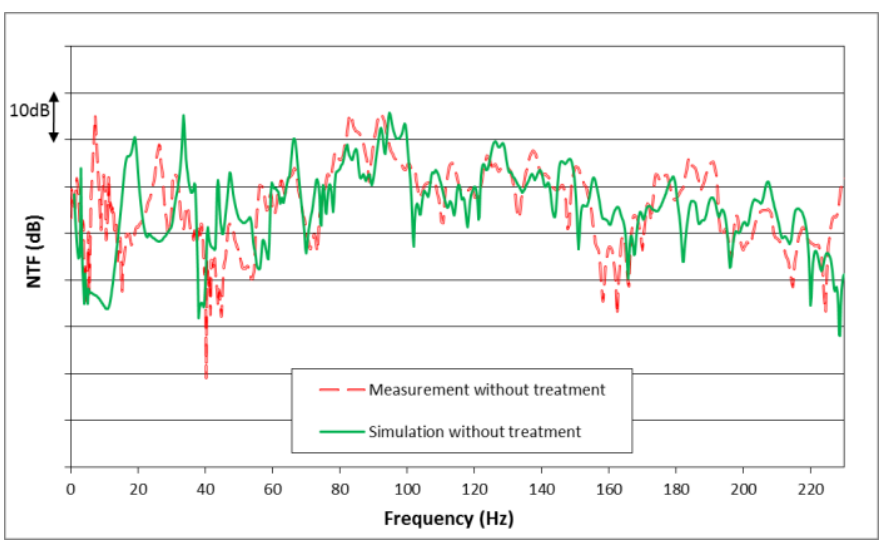

Figure 14: Measured and simulated NTF between the front left anchorage point and the driver's ear

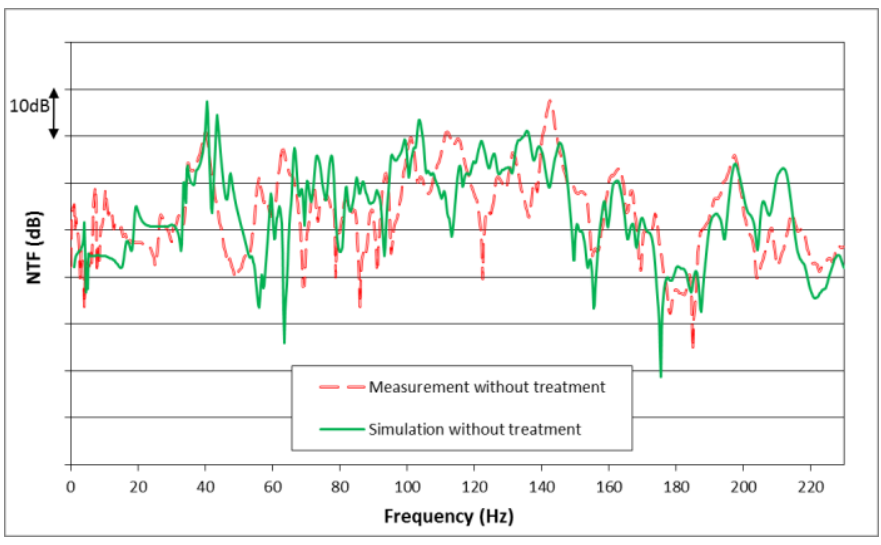

Figure 15: Measured and simulated NTF between a point on the rear wall and the driver's ear

Main discrepancies can be observed below $50 \mathrm{~Hz}$. This is below the first cavity mode and corresponds to the frequency range where the acoustic treatments have no effect (see Figure 8). A possible explanation lies in the handle of rigid body modes but this is not verified since correlation efforts are put on frequencies above $50 \mathrm{~Hz}$.

\section{Results with a single acoustic treatment}

In a first step, the effect of the floormat and the rear trim panel taken independently is observed.

\section{Floormats only}

For a cabin equipped with the floormats only, the NTF from the tunnel point to the driver's ear is plot Figure 16. On the same plot, the noise reduction brought by the floormats is added both as measured 
and simulated. This allows freeing the results from the discrepancies of the bare model.

Measured and simulated curves show similar trends and levels. When looking at the noise reduction between the bare cabin and the cabin equipped with floormats, the noise reduction is correctly simulated. Levels of noise reduction are close. They are sometimes shifted in frequency. This is inherited from the frequency shift between measured and simulated modes in the bare model as the floormats mainly damp the panel modes.

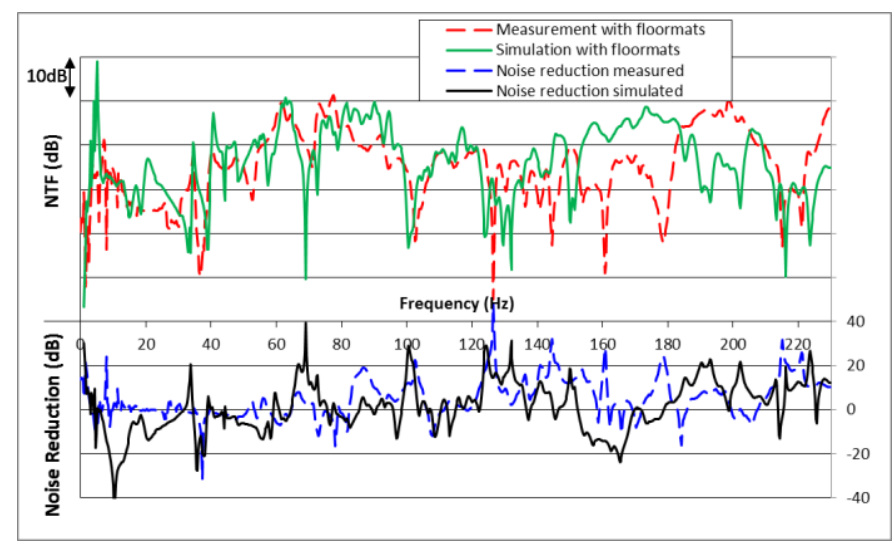

Figure 16: Measured and simulated NTF and noise reduction in presence of the floormats. Excitation from the tunnel point and delta

Such floormats made of a thick layer of polyurethane and a heavy layer of rubber have a resonance frequency. Noise from the panel is generally amplified at this frequency. For the floormats considered in this study, the resonance occurs around $160 \mathrm{~Hz}$. The drop of noise reduction around this frequency both in measurement and simulation shows that the simulation properly models this phenomenon.

\section{Rear trim panel only}

For a cabin equipped with the rear trim panel, the NTFs from the rear wall point to the driver's ear are shown Figure 17.

Measured and simulated curves show similar trend and levels. The noise reduction given by the rear trim panel only is correctly simulated. This effect can be mainly observed when frequency increases $(>180 \mathrm{~Hz})$. The over-estimated sound reduction at some frequencies $(135 \mathrm{~Hz} / 145 \mathrm{~Hz})$ is due to anti-resonance phenomena.

Below $50 \mathrm{~Hz}$, although the effect of the panel is negligible, simulation shows noise reduction. This difference is not explained so far but the possible interaction of rigid body modes with the treatment is not excluded.

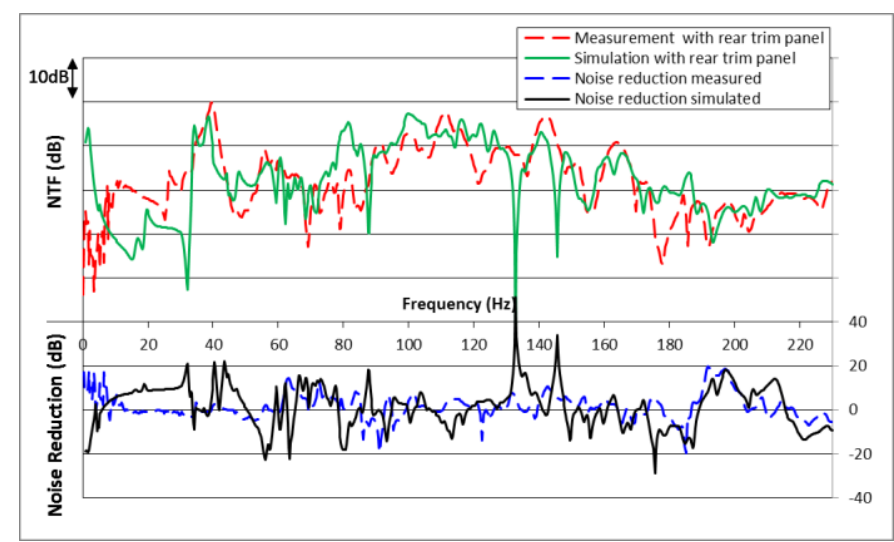

Figure 17: Measured and simulated NTF and noise reduction in presence of the rear trim panel. Excitation from a rear wall point

\section{Results with all acoustic treatments}

For a cabin equipped with all the acoustic treatments (headliner, rear trim panel and all floormats), the NTFs from the anchorage points of the cabin to the driver's ear are analysed. Figure 18 and Figure 19 show comparison of the NTF and the Mean NTF (average from 18 microphones).

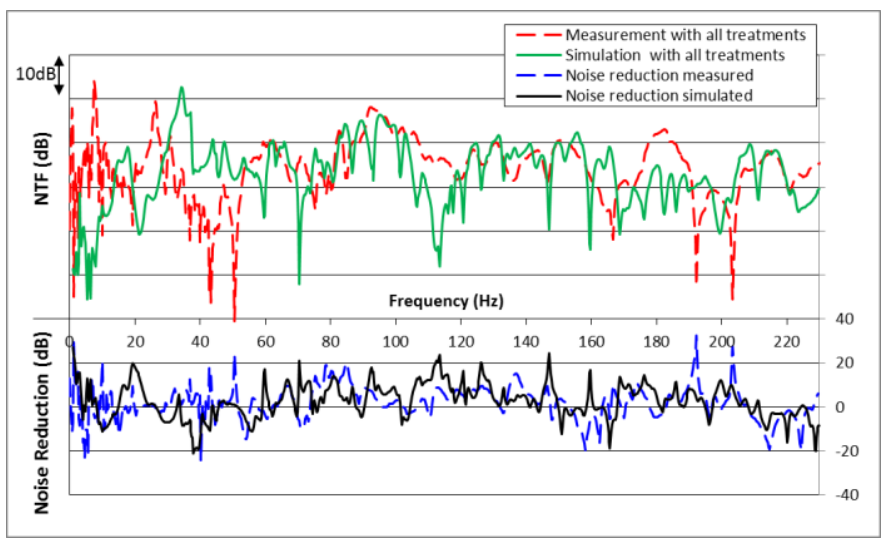

Figure 18: Measured and simulated NTF and noise reduction in presence of all the acoustic treatments. Excitation from the front left anchorage point.

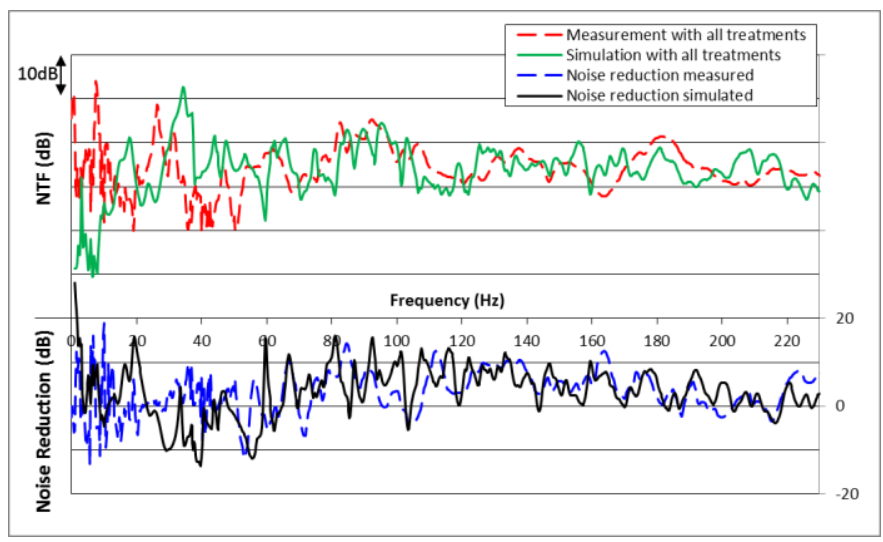

Figure 19: Measured and simulated Mean NTF (average 18 microphones) and noise reduction in presence of all the acoustic treatments. Excitation from the front left anchorage point

Page 7 of 8 
The correlation between the measurements and the simulation is satisfying. The noise reduction given by the treatments is properly simulated which is the main objective of the works.

By comparing with the results from the configurations without treatments and with a single treatment, it is possible to analyse the treatment responsible for a particular effect or that is the cause of a discrepancies at a given frequency.

This level of correlation of the noise reduction between measurement and simulation validates the method and the modelling. One can be confident that the behaviour of the treatments is correctly modelled. However, the quality of the results below $50 \mathrm{~Hz}$ remains low, need to be explained and improved.

\section{Conclusion}

The challenge of vehicle weight reduction requires accurate methods to predict its vibro-acoustic behavior including the acoustic treatments.

The method presented in this paper aims at predicting the vibroacoustic response of the cabin including the acoustic treatments while using a modal scheme $(0-300 \mathrm{~Hz})$.

A measurement campaign is performed to get reference data to compare with numerical results.

The FEM model without any treatment is first correlated to the measurements. This is an important step to limit the errors on the models considering the treatments, as they are based on this first model. A good level of correlation is obtained both on VTF and NTF indicators.

As final result, the FEM model with all the treatments is compared to the measurements. Satisfying correlation is obtained on the VTF and NTF and very similar levels of noise reduction are obtained above $50 \mathrm{~Hz}$

Although the modelling needs to be improved below $50 \mathrm{~Hz}$, the method is considered as validated for the analysis of future weight reductions of truck cabins.

\section{References}

1. Maxit, L., Guyader, J.L., Ege, K., Gerges, Y. et al., "SmEdA vibro-acoustic modeling of a trimmed truck cab in the midfrequency range", SAE Technical Paper 2018-01-1506, 2018.

2. Coyette, J.-P.,Lielens, G., Van den Nieuwenhof, B., Bertolini, C. and al.,"From Body in White to Trimmed Body Models in the Low Frequency Range: a New Modeling Approach", SAE Technical Paper 2007-01-2340, 2007, doi:10.4271/2007-01$\underline{2340}$.

3. Van den Nieuwenhof, B., Lielens, G., Coyette, J.P., Acher, F. and al., "Efficient analysis of large trimmed configurations using modal approaches", ISMA conference on Noise and Vibration Engineering, (Leuven, Belgium), 2008.

4. Bertolini, C., Gaudino, C., Caprioli, D., Misaji, K. et al., "FE Analysis of a Partially Trimmed Vehicle using Poroelastic Finite Elements Based on Biot's Theory," SAE Technical Paper 200701-2330, 2007, doi:10.4271/2007-01-2330.
5. Johnson, D. L., Koplik, J., Dashen R., "Theory of dynamic permeability and tortuosity in fluid saturated porous media", $J$. Fluid Mech., 176:379-402, 1987.

6. Champoux, Y., Allard, J.-F., "Dynamic tortuosity and bulk modulus in air-saturated porous media", J. Appl. Phys., 70:1975-1979, 1991.

7. Beranek, L. L., "Acoustic impedance of porous materials", $J$. Acoust. Soc. Am., 13:248-260, 1942.

\section{Contact Information}

Fabien Acher - fabien.acher@volvo.com Volvo GTT, Lyon, France

\section{Acknowledgments}

The authors acknowledge Oleg Danilov (Volvo GTT), for his valuable advices, the FFT / MSC Software experts for their support in development of the model and the Matelys Company for the collaboration in the characterization of the porous materials.

The measurement works were co-funded by the French government (FUI 12 - Fonds Unique Interministériel) and European Union (FEDER - Fonds européen de développement régional). It was carried out in the framework of the LabEx CeLyA ("Centre Lyonnais d'Acoustique", ANR-10-LABX-60) and the research project CLIC ("City Lightweight Innovative Cab") labelled by LUTB cluster (Lyon Urban Truck and Bus), in partnership with Renault Trucks, ArcelorMittal, ACOEM, ALTRAN, FEMTO-ST (Univ. de Franche-Comté) and LVA (INSA de Lyon).

\section{Definitions/Abbreviations}

$\begin{array}{ll}\text { NTF } & \text { Noise Transfer Function } \\ \text { VTF } & \text { Vibration Transfer Function. } \\ \text { FEM } & \text { Finite Element Method. }\end{array}$

